\title{
Acoustic-gravity waves in the atmosphere: from Zakharov equations to wave-kinetics
}

\author{
J. T. Mendonca and Lennart Stenflo
}

\section{Linköping University Post Print}

\section{Tweet}

N.B.: When citing this work, cite the original article.

Original Publication:

J. T. Mendonca and Lennart Stenflo, Acoustic-gravity waves in the atmosphere: from Zakharov equations to wave-kinetics, 2015, Physica Scripta, (90), 5, 055001.

http://dx.doi.org/10.1088/0031-8949/90/5/055001

Copyright: IOP Publishing: Hybrid Open Access

http://www.iop.org/

Postprint available at: Linköping University Electronic Press

http://urn.kb.se/resolve?urn=urn:nbn:se:liu:diva-120293 
Acoustic-gravity waves in the atmosphere: from Zakharov equations to wave-kinetics

This content has been downloaded from IOPscience. Please scroll down to see the full text. 2015 Phys. Scr. 90055001

(http://iopscience.iop.org/1402-4896/90/5/055001)

View the table of contents for this issue, or go to the journal homepage for more

Download details:

IP Address: 130.236.83.116

This content was downloaded on 02/09/2015 at 12:56

Please note that terms and conditions apply. 


\title{
Acoustic-gravity waves in the atmosphere: from Zakharov equations to wave-kinetics
}

\author{
J T Mendonça ${ }^{1,3}$ and L Stenflo ${ }^{2}$ \\ ${ }^{1}$ IPFN, Instituto Superior Técnico, 1049-001 Lisboa, Portugal \\ ${ }^{2}$ Department of Physics, Linkoping University, SE-58183 Linkoping, Sweden \\ E-mail: titomend@ist.utl.pt and Lennart.Stenflo@physics.umu.se
}

Received 14 January 2015

Accepted for publication 19 February 2015

Published 1 April 2015

\begin{abstract}
We develop a wave-kinetic description of acoustic-gravity (AG) waves in the atmosphere. In our paper the high frequency spectrum of waves is described as a gas of quasi-particles. Starting from the Zakharov-type of equations, where coupling between fast and slow density perturbations is considered, we derive the corresponding wave-kinetic equations, written in terms of an appropriate Wigner function. This provides an alternative description for the nonlinear interaction between the two dispersion branches of the AG waves.
\end{abstract}

Keywords: acoustic-gravity waves, Zakharov equations, Wigner function

\section{Introduction}

Acoustic-gravity (AG) waves are of fundamental importance for the understanding of the Earth's atmosphere. They have been studied by several authors, in both linear and nonlinear regimes [1-7].

It is well known that the AG waves have two branches, corresponding to low and high frequency modes. Waves in the lower frequency branch are usually called internal gravity waves. Their mutual interaction can be described by coupled equations similar to the acclaimed Zakharov equations [8], which were first derived to study electrostatic turbulence in non-magnetized plasmas [9, 10].

In a recent work [11], we have proposed a new approach to atmospheric turbulence, based on the wave-kinetic description of the oscillation spectrum. This led to a two-fluid model of the atmosphere, similar to that first proposed by Tisza and London for turbulent superfluids, and then developed by Landau and others [12]. In this case, the two fluids are, the atmosphere itself and the gas of quasi-particles describing the wave spectrum. In this work, we had mainly focused on the destabilization of zonal flows by internal gravity waves.

Here we extend the two-fluid description of the atmospheric turbulence, by considering the nonlinear coupling

Author to whom any correspondence should be addressed. between the high frequency branch of the AG waves with low frequency perturbations. In particular, we focus on the Zakharov-type of equations, as derived by [8]. The purpose of the present work is to show that wave-kinetic equations for the atmosphere can be directly derived from these Zakharov equations, thus establishing a clear link between the two different approaches. We also show that the wave-kinetic equations present some advantages with respect to the Zakharov equations, because they reveal the existence of Landau quasi-particle resonances, which play a relevant role on the coupling between high and low frequency waves.

The content of this paper is the following. In section 2 we start with the fluid equations for the atmosphere, establish the linear dispersion relations and state the Zakharov-type of equations describing the nonlinear fluid response. In section 3 we derive the corresponding wave-kinetic equation, that describes the evolution of the density correlations and of the corresponding Wigner function. In the geometric optics limit, to be specified, this equation reduces to a Vlasov equation for the high frequency quasi-particles, which are indeed a particular class of phonons. In section 4 we illustrate the interest of this new approach by deriving a nonlinear dispersion relation for the internal gravity waves, in the presence of an arbitrary turbulent spectrum. Finally, in section 5, we draw some conclusions. 


\section{Zakharov equations}

The Earth atmosphere can be described by the following fluid equations, relating the velocity $\mathbf{v}$, and density $\rho$, as

$$
\frac{\partial \rho}{\partial t}+\nabla \cdot(\rho \mathbf{v})=0
$$

and

$$
\left(\frac{\partial}{\partial t}+\mathbf{v} \cdot \nabla\right) \mathbf{v}=\mathbf{g}-\frac{\nabla P}{\rho},
$$

where $\mathbf{g}=-g \mathbf{e}_{z}$ is the gravitational acceleration. The gas pressure $P$ can be determined by the equation of state

$$
\left(\frac{\partial}{\partial t}+\mathbf{v} \cdot \nabla\right)\left(\rho^{-\gamma} P\right)=0
$$

where $\gamma$ is the ratio between specific heats. We assume that, in equilibrium, the fluid density decays with the altitude, according to

$$
\rho_{0}(z)=\rho_{0}(0) \exp \left(-2 k_{0} z\right), \quad k_{0}=\frac{\gamma g}{2 c_{s}^{2}},
$$

where $c_{s}$ is the sound speed. The scale length of the atmosphere is therefore given by $H=1 / 2 k_{0}$.

We now introduce the density perturbations $\tilde{\rho}=\rho-\rho_{0}(z)$, and assume that $|\tilde{\rho}| \ll \rho_{0}$. Linearizing equations (1)-(3), we can derive an evolution equation for the small perturbations, of the form

$$
\left[\frac{\partial^{4}}{\partial t^{4}}+\left(\omega_{a}^{2}-c_{s}^{2} \nabla^{2}\right) \frac{\partial^{2}}{\partial t^{2}}-\omega_{g}^{2} c_{s}^{2} \nabla_{\perp}^{2}\right] \tilde{n}=0,
$$

where we have introduced a new variable $\tilde{n}=\tilde{\rho} / \sqrt{\rho_{0}}$, and used the perpendicular Laplacian operator $\nabla_{\perp}^{2} \equiv \partial^{2} / \partial x^{2}+\partial^{2} / \partial y^{2}$. We have also defined two characteristic frequencies, $\omega_{a}$ and $\omega_{g}$, such that

$$
\omega_{a}^{2}=k_{0}^{2} c_{s}^{2}, \quad \omega_{g}^{2}=\left(1-\frac{1}{\gamma}\right) 2 g k_{0} .
$$

These two frequencies define two distinct wave dispersion branches, as discussed next. The ratio between them is $\left(\omega_{g} / \omega_{a}\right)^{2}=4(\gamma-1) / \gamma^{2}$. It can be assumed that the value of $\gamma$ is only slightly larger than unity, thus making the two characteristic frequencies quite distinct from each other. Assuming that the density perturbations behave in space and time as $\tilde{n} \propto \exp (\mathbf{i k} \cdot \mathbf{r}-\mathbf{i} \omega t)$, where $\omega$ is the wave frequency and $\mathbf{k}$ its wavevector, we obtain the dispersion equation [3]

$$
\omega^{2}\left(\omega^{2}-\omega_{a}^{2}\right)-c_{s}^{2}\left[\left(\omega^{2}-\omega_{g}^{2}\right) k^{2}+\omega_{g}^{2} k_{z}^{2}\right]=0 .
$$

This expression reveals the existence of two distinct dispersion branches, with frequencies $\omega \simeq \omega_{h}$ and $\omega \simeq \Omega$, which can be represented by the following approximate relations

$$
\omega_{h}^{2}=\omega_{a}^{2}+c_{s}^{2} k^{2}, \quad \Omega^{2}=\frac{\omega_{g}^{2} k_{\perp}^{2}}{k^{2}+k_{0}^{2}} .
$$

Waves satisfying the low frequency dispersion branch are usually called internal gravity waves. These two wave branches can be coupled nonlinearly, and a nonlinear analysis of the atmosphere equations (1)-(3) shows that the high frequency perturbations, $\tilde{n}_{h}$, satisfying equation (7), can be described by the following evolution equation [8]

$$
\sqrt{\rho_{0}}\left[\frac{\partial^{2}}{\partial t^{2}}+c_{s}^{2}\left(k_{0}^{2}-\nabla^{2}\right)\right] \frac{\partial M_{h}}{\partial t}=S_{h},
$$

where the high frequency variables are now defined as

$$
M_{h}=\int^{t} \tilde{n}_{h}\left(t^{\prime}\right) \mathrm{d} t^{\prime}, \quad \mathbf{v}_{h}=-c_{s}^{2} \nabla \frac{M_{h}}{\sqrt{\rho_{0}}},
$$

and the source term is determined by

$$
S_{h}=\nabla \cdot\left[\mathbf{v}_{h} \cdot \nabla \mathbf{J}_{l}+\mathbf{J}_{l}\left(\nabla \cdot \mathbf{v}_{h}\right)+\left(\mathbf{J}_{l} \cdot \nabla\right) \mathbf{v}_{h}\right] .
$$

Here, the low frequency variable is the current $\mathbf{J}_{l}=\rho_{0} \mathbf{v}_{l}$. Its temporal evolution can be described by

$$
\frac{\partial}{\partial t} \mathbf{J}_{l}=-\frac{k_{0}}{2} c_{s}^{4}\left(\nabla_{\perp} \frac{\partial}{\partial z}-\mathbf{e}_{z} \nabla_{\perp}^{2}\right)\left\langle M_{h}^{2}\right\rangle .
$$

Equations (9) and (12) can be seen as the Zakharov-type of equations describing the nonlinear coupling between the low and the high frequency branches of the AG waves in the atmosphere. This coupling is due to the ponderomotive force produced by the high frequency oscillations, as represented in equation (12). It will be shown, in the next section, that such a coupling can also be described by an equivalent set of wavekinetic equations, where the high frequency waves are described in terms of quasi-particles.

\section{Wave-kinetic equations}

Let us go back to the wave equation for high frequency waves (9), and derive the equivalent wave-kinetic equation, which determines the evolution of the density auto-correlations. For that purpose, let us assume with generality that the high frequency perturbations can be represented by a superposition of modes with amplitudes $M_{k}$, as defined by the Fourier integral

$$
M_{h}(\mathbf{r}, t)=\int M_{k} \exp (\mathrm{ik} \cdot \mathbf{r}-\mathrm{i} \omega t) \frac{\mathrm{d} \mathbf{k}}{(2 \pi)^{3}},
$$

where $\omega \equiv \omega(\mathbf{k})$ satisfies a given dispersion relation, to be specified below. From equation (9), we obtain, for each frequency mode

$$
\begin{aligned}
{\left[\omega^{2}-\left(\omega_{a}^{2}+k^{2} c_{s}^{2}\right)\right] M_{k}=} & \frac{\mathbf{k}}{\omega \sqrt{\rho_{0}}} \cdot\left[\mathbf{v}_{k} \cdot \nabla \mathbf{J}_{l}\right. \\
& \left.+\mathbf{J}_{l}\left(\mathrm{ik} \cdot \mathbf{v}_{k}\right)+\mathrm{i}\left(\mathbf{J}_{l} \cdot \mathbf{k}\right) \mathbf{v}_{k}\right],
\end{aligned}
$$

with

$$
\mathbf{v}_{k}=-\frac{c_{s}^{2}}{\sqrt{\rho_{0}}}\left(\mathrm{ik}+k_{0} \mathbf{e}_{z}\right) M_{k}
$$


Replacing this in equation (14), we obtain

$$
\omega^{2}-\left(\omega_{a}^{2}+k^{2} c_{s}^{2}\right)=-\frac{c_{s}^{2}}{\rho_{0}} \mathcal{L}_{k} \mathbf{J}_{l}(\mathbf{r}, t),
$$

where the nonlinear coupling operator is defined by

$$
\mathcal{L}_{k}=\frac{\mathbf{k}}{\omega} \cdot\left[\left(\mathrm{ik}+k_{0} \mathbf{e}_{z}\right) \cdot(\mathrm{ik}+\nabla)+\left(\mathrm{i} k_{0} k_{z}-k^{2}\right)\right] .
$$

In the absence of this nonlinear coupling, equation (16) reduces to the linear dispersion relation for the high frequency branch of equation (8). In the presence of coupling, this can be seen as a local dispersion relation, where the linear properties are slightly modified by the slowly varying current $\mathbf{J}_{l}(\mathbf{r}, t)$. But it should also be noted that the nonlinear coupling introduces new effects, such as an energy transfer between the different ranges of the AG waves. These qualitative changes imply that the above description needs to be refined in order to account for this energy transfer. In particular, the amplitudes of both $M_{k}$ and $\mathbf{J}_{l}$ will evolve slowly in time, and cannot be assumed constant. This slow time evolution can be introduced by going back to equation (14) and replace $\omega^{2}$ by the operator $\left(\omega^{2}+2 \mathrm{i} \omega \partial / \partial t\right)$. The total time evolution of the high frequency mode $M_{k}(t)$ can then be determined by the equation

$$
\left(\frac{\partial}{\partial t}+\mathrm{i} \omega\right) M_{k}(t)+\int \frac{\mathrm{d} \mathbf{q}}{(2 \pi)^{3}} \mathcal{Q}_{k}(\mathbf{q}) J_{q}(t) M_{k^{\prime}}(t)=0,
$$

where $\mathbf{J}_{q}(t)$ are the spatial Fourier components of the nonlinear current $\mathbf{J}_{l}(\mathbf{r}, t)$, and we have introduced the wave vector $\mathbf{k}^{\prime}=\mathbf{k}-\mathbf{q}$. We have also used the expression

$$
\begin{aligned}
\mathcal{Q}_{k}(\mathbf{q})= & \frac{\mathrm{i} c_{s}^{2}}{2 \omega^{2} \rho_{0}} \mathbf{k} \cdot\left[\left(\mathrm{i} k_{0} \mathbf{e}_{z}-\mathbf{k}^{\prime}\right)\left(\mathbf{k} \cdot \mathbf{e}_{q}\right)\right. \\
& \left.+\mathbf{e}_{q}\left(\mathrm{i} k_{0} k_{z}^{\prime}-k^{\prime 2}\right)\right] .
\end{aligned}
$$

Here, we have introduced the unit vector $\mathbf{e}_{q} \equiv \mathbf{J}_{q} /\left|J_{q}\right|$. Note that, in general, the typical acoustic wavelengths are much larger than the height of the atmosphere, $|k| \gg k_{0}$, and also $\mathbf{k} \simeq \mathbf{k}^{\prime}$. We can now use the standard Wigner-Moyal procedure, in the way described by [14]. Introducing the Wigner function for the high frequency perturbations, as

$$
\begin{aligned}
W(\mathbf{r}, \mathbf{k}, t)= & \int M_{h}(\mathbf{r}-\mathbf{s} / 2, t) M_{h}^{*}(\mathbf{r}+\mathbf{s} / 2, t) \\
& \times \exp (\mathbf{i k} \cdot \mathbf{s}) \mathrm{d} \mathbf{s},
\end{aligned}
$$

and following such a proceedure, we obtain the wave-kinetic equation

$$
\begin{aligned}
\left(\frac{\partial}{\partial t}+\mathbf{v}_{k} \cdot \nabla\right) W= & -\int \frac{\mathrm{d} \mathbf{q}}{(2 \pi)^{3}} \mathcal{Q}_{k}(\mathbf{q}) J_{q}(t)\left[W^{-}-W^{+}\right] \\
& \times \exp (\mathrm{iq} \cdot \mathbf{r}),
\end{aligned}
$$

where $W \equiv W(\mathbf{r}, \mathbf{k}, t)$, and $W^{ \pm}=W(\mathbf{r}, \mathbf{k} \pm \mathbf{q} / 2, t)$. Here we have used the group velocity, $\mathbf{v}_{k}=\partial \omega / \partial \mathbf{k}=c_{s}^{2} \mathbf{k} / \omega$. This equation describes the space and time evolution of the high frequency quasi-distribution $W$, in the presence of low frequency perturbations with spectral components $J_{q}(t)$. It should be noticed that $W$ reduces to a distribution function for the high frequency atmospheric phonons, in the limit of geometric optics approximation. But, in general, it can be negative and complex, when such an approximation is not valid.

The geometric optics approximation is valid when the low frequency perturbations of the medium, described by the q spectrum, have a typical scale length much larger than the high frequency oscillations described by the Wigner function $W$, or to be more specific, when $|k| \gg|q|$. In that limit, the difference $\left[W^{-}-W^{+}\right]$can be developed in series, and to the lowest order, equation (21) reduces to

$$
\left(\frac{\partial}{\partial t}+\mathbf{v}_{k} \cdot \nabla+\mathbf{F}_{k} \cdot \frac{\partial}{\partial \mathbf{k}}\right) W=0,
$$

where the nonlinear force $\mathbf{F}_{k}$ acting on the phonons is determined by

$$
\mathbf{F}_{k}=-\nabla V_{k}, \quad V_{k}(\mathbf{r}, t)=\mathcal{Q}_{k}(\mathbf{q}) J_{q}(t) \exp (\mathrm{iq} \cdot \mathbf{r}) .
$$

The new quantity $V_{k}$ is obviously a nonlinear potential. This new equation takes the form of a kinetic Vlasov equation, which can also be established for photons in optics, and for a large class of quasi-particles in classical and quantum fluids [13].

\section{Stability of large scale perturbations}

In order to complete the above description, we only need to state the evolution equation for the slowly varying current $J_{l}(t)$, in terms of the Wigner function. This can easily be done by rewriting equation (12) in the form

$$
\frac{\partial}{\partial t} \mathbf{J}_{l}=-\frac{k_{0}}{2} c_{s}^{4}\left(\nabla_{\perp} \frac{\partial}{\partial z}-\mathbf{e}_{z} \nabla_{\perp}^{2}\right) \int W(\mathbf{r}, \mathbf{k}, t) \frac{\mathrm{d} \mathbf{k}}{(2 \pi)^{3}} .
$$

Equations (21) and (24) are the wave-kinetic equivalent to the Zakharov equations (9) and (12), as they exactly convey the same physical information. They can provide an alternative description of the nonlinear coupling between high frequency and low frequency wave perturbations in the atmosphere. A similar approach was already used by us to describe the destabilization of zonal flows by internal gravity waves [11].

To illustrate the interest of the present approach, we consider the case of a single low frequency mode, such that $\mathbf{J}_{l}(\mathbf{r}, t)=\mathbf{J}_{q} \exp (\mathrm{iq} \cdot \mathbf{r}-\mathrm{i} \Omega t)$. We also assume that the mode approximately satisfies the low frequency dispersion branch of equation (8). This allow us to write

$$
\Omega^{2} \sim \Omega_{q}^{2} \equiv \frac{\omega_{g}^{2} q_{\perp}^{2}}{q^{2}+k_{0}^{2}} .
$$

To be consistent with such an assumption, we include the total temporal evolution of the mode $\mathbf{J}_{q}$ in equation (24), 
which becomes

$$
\begin{aligned}
\left(\frac{\partial}{\partial t}+\mathrm{i} \Omega_{q}\right) \mathbf{J}_{p}= & \frac{k_{0}}{2} c_{s}^{4}\left(\mathbf{q}_{\perp} q_{z}-\mathbf{e}_{z} q_{\perp}^{2}\right) \\
& \int W_{q}(\mathbf{r}, \mathbf{k}) \frac{\mathrm{d} \mathbf{k}}{(2 \pi)^{3}} .
\end{aligned}
$$

Here $W_{q}(\mathbf{r}, \mathbf{k})$ represents the low frequency modulations induced on the quasi-distribution $W(\mathbf{r}, \mathbf{k}, t)$ by the slowly varying current. In the absence of nonlinear coupling, this equation would simply reduce to the identity $\Omega=\Omega_{q}$. Retaining the nonlinear correction, we need to determine the value of the perturbation $W_{q}$. For that purpose we linearize the wave-kinetic equation (21), which leads to

$$
W_{q}=Q_{k}(\mathbf{q}) \frac{\left[W_{0}^{-}-W_{0}^{+}\right]}{\left(\Omega-\mathbf{q} \cdot \mathbf{v}_{k}\right)} J_{q},
$$

where $W_{0}$ is the equilibrium value of the quasi-distribution. Replacing this in equation (26) we can then obtain a modified dispersion relation of the form

$$
\begin{aligned}
1 & -\frac{\Omega_{p}}{\Omega}-\mathrm{i} \frac{k_{0} c_{s}^{4}}{2 \Omega}\left(q_{z} \mathbf{q}_{\perp}-q_{\perp}^{2} \mathbf{e}_{z}\right) \\
& \cdot \mathbf{e}_{q} \int Q_{k}(\mathbf{q}) \frac{\left[W_{0}^{-}-W_{0}^{+}\right]}{\left(\Omega-\mathbf{q} \cdot \mathbf{v}_{k}\right)} \frac{\mathrm{d} \mathbf{k}}{(2 \pi)^{3}}=0 .
\end{aligned}
$$

It should be noticed that $\mathbf{e}_{q}=\left[\left(\mathbf{q}_{\perp} / q_{\perp}\right) q_{z}-\mathbf{e}_{z} q_{\perp}\right] / q$. This means that the nonlinear currents are always perpendicular to the perturbation wavevector, $\left(\mathbf{e}_{q} \cdot \mathbf{q}\right)=0$. Equation (28) determines the nonlinear dispersion of a single mode with frequency $\Omega$ and wavevector $\mathbf{q}$, in the presence of an arbitrary spectrum of high frequency oscillations. We notice the appearance of a Landau resonance in the nonlinear susceptibility term, which defines a privileged channel for energy transfer between high and low frequency oscillations. This occurs when the group velocity $\mathbf{v}_{k}$ of the high frequency quasi-particles become equal to the phase velocity of the low frequency oscillations.

\section{Conclusions}

We have applied the wave-kinetic description to nonlinear wave coupling in the atmosphere. We have shown that the spectrum of AG waves can be seen as a gas of quasi-particles. These quasi-particles are indeed a particular class of phonons. Starting from the Zakharov-type of equations describing the nonlinear evolution of AG waves, and using the standard Wigner-Moyal procedure, we have derived the corresponding wave-kinetic equations. We have therefore established an equivalence between the Zakharov and the wave-kinetic descriptions.

To illustrate the interest of this new formulation, we have also derived the dispersion relation of internal gravity waves in the presence of an arbitrary spectrum of high frequency atmospheric turbulence. This is formally analogous to the dispersion relation of zonal flows in a spectrum of internal gravity waves, as derived in [11], but with a clearly distinct physical meaning. In a sense, the present paper completes the wave-kinetic description of the atmosphere, started by our previous work.

The Zakharov and the wave-kinetic approach, being nearly equivalent, can provide two complementary views of the atmospheric turbulence. In particular, the Zakharov approach seems to be more adequate for the derivation of soliton solutions. In contrast, the wake-kinetic approach explicitly states a Landau resonance between the low frequency waves and the high frequency quasi-particles. It also seems more adapted to derive nonlinear dispersion relations, and to describe the subtle dependence of the growth rates with the turbulence spectrum.

\section{Acknowledgments}

This research was partially supported by the International Space Science Institute in Bern (Switzerland) through the grant 'Large-scale vortices and zonal winds in planetary atmospheres/ionospheres: theory versus observations'.

\section{References}

[1] Hines C O 1960 Can. J. Phys. 381441

[2] Yeh K C and Liu C H 1970 Radio Sci. 539

[3] Dysthe K B, Jurén C and Stenflo L 1974 Phys. Scr. 9226

[4] Stenflo L 1987 Phys. Fluids 303297

[5] Widdel H U, Bittner M and Hoppe U P 1994 J. Atmos. Sol.Terr. Phys. 561779

[6] Kshevetskii S P and Gavrilov N M 2005 J. Atmos. Sol.-Terr. Phys. 671014

[7] Huang K M, Zhang S D and Yi F 2011 J. Atmos. Sol.-Terr. Phys. 732429

[8] Stenflo L 1986 Phys. Scr. 33156

[9] Zakharov V E 1972 Sov. Phys. JETP 35908

[10] Goldman M V 1984 Rev. Mod. Phys. 56709

[11] Mendonça J T, Onishchenko O G, Pokhotelov O A and Stenflo L 2014 Phys. Scr. 89125004

[12] Leggett A J 2006 Quantum Liquids (Oxford: Oxford University Press)

[13] Mendonça J T 2014 Eur. Phys. J. D 6879

[14] Mendonça J T and Hyzanidis K 2011 Phys. Plasmas 18 112306 